\title{
Stark effect or coverage dependence? Disentangling the EC-SEIRAS vibrational shift of sulfate on Au(111)
}

Cite as: J. Chem. Phys. 150, 041709 (2019); https://doi.org/10.1063/1.5047941

Submitted: 10 July 2018 . Accepted: 21 August 2018 . Published Online: 05 December 2018

Jonas H. K. Pfisterer, Ulmas E. Zhumaev, William Cheuquepan, Juan M. Feliu, and Katrin F. Domke (D)

\section{COLLECTIONS}

Paper published as part of the special topic on Interfacial Electrochemistry and Photo(electro)catalysis
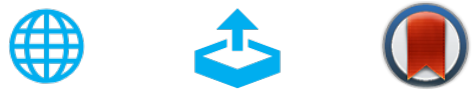

View Online

Export Citation

\section{ARTICLES YOU MAY BE INTERESTED IN}

Multistep and multiscale electron transfer and localization dynamics at a model electrolyte/metal interface

The Journal of Chemical Physics 150, 041702 (2019); https://doi.org/10.1063/1.5047033

Grand-canonical approach to density functional theory of electrocatalytic systems:

Thermodynamics of solid-liquid interfaces at constant ion and electrode potentials

The Journal of Chemical Physics 150, 041706 (2019); https://doi.org/10.1063/1.5047829

Glycerol electrooxidation on Pd modified Au surfaces in alkaline media: Effect of the deposition method

The Journal of Chemical Physics 150, 041703 (2019); https://doi.org/10.1063/1.5048489

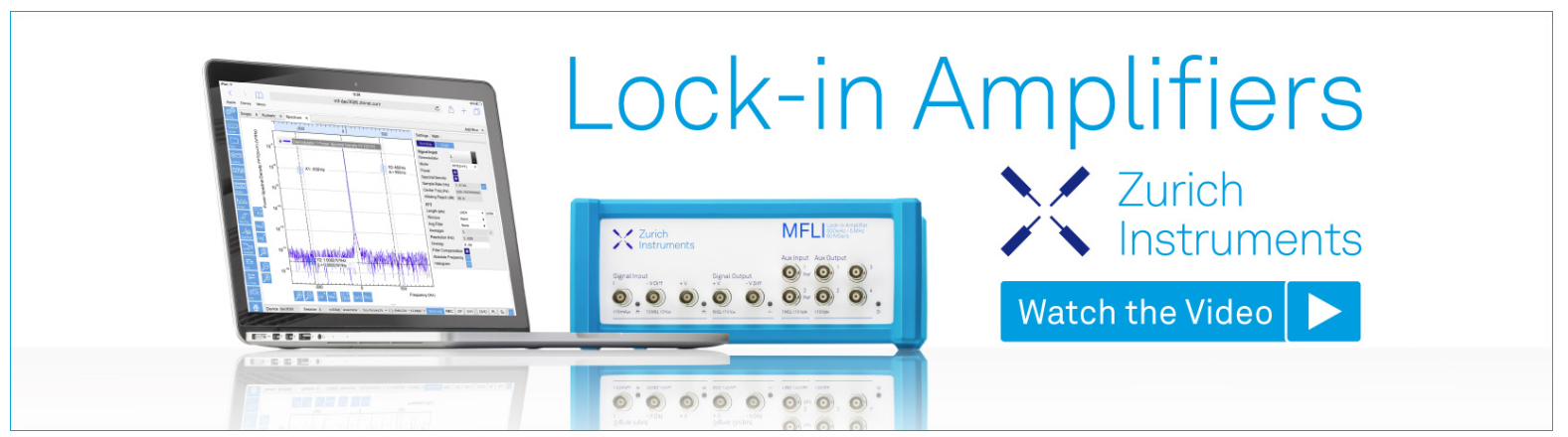




\title{
Stark effect or coverage dependence? Disentangling the EC-SEIRAS vibrational shift of sulfate on Au(111)
}

\author{
Cite as: J. Chem. Phys. 150, 041709 (2019); doi: 10.1063/1.5047941 \\ Submitted: 10 July 2018 - Accepted: 21 August 2018 • \\ Published Online: 5 December 2018
}

Jonas H. K. Pfisterer, ${ }^{1}$ Ulmas E. Zhumaev, ' William Cheuquepan, ${ }^{2}$ Juan M. Feliu, ${ }^{2}$ and Katrin F. Domke ${ }^{1, a)}$ (i)

\author{
AFFILIATIONS \\ ${ }^{1}$ Molecular Spectroscopy Department, Max Planck Institute for Polymer Research, Ackermannweg 10, 55128 Mainz, Germany \\ ${ }^{2}$ Instituto de Electroquímica, Universidad de Alicante, Apdo. 99, 03080 Alicante, Spain \\ a) Author to whom correspondence should be addressed: domke@mpip-mainz.mpg.de. Tel: +49 (0) 6131 379-476.
}

\begin{abstract}
Infrared spectroscopy is a widely employed analytical tool in (electrochemical) surface science as the spectra contain a wealth of information about the interaction of interfacial adsorbates with their environment. Separating and quantifying individual contributions, for example, of co-adsorbates, the substrate or electric field effects, on the overall spectral response, however, is often non-trivial as the various interactions manifest themselves in similar spectral behavior. Here, we present an experimental approach to differentiate between and quantify potential-induced coverage dependence and field-related Stark effects observed in a sulfate band shift of $93.5 \pm 1.5 \mathrm{~cm}^{-1} / \mathrm{V}$ in electrochemical infrared spectra of the showcase sulfate/Au(111) interface. In combination with a simple linear model equation used to describe the potential-induced peak shift of the sulfate stretch vibration, we determine the coverage dependence contribution to be $15.6 \pm 1.2 \mathrm{~cm}^{-1} / \theta_{\mathrm{SO}}$ and the Stark effect to amount to $75.6 \pm 2.7 \mathrm{~cm}^{-1} / \mathrm{V}$. Our work provides a novel route to gain fundamental insight into interfacial adsorbate interactions in electrochemical surface science.
\end{abstract}

Published under license by AIP Publishing. https://doi.org/1 0.1 063/1.5047941

\section{INTRODUCTION}

Infrared absorption spectroscopy (IRAS) is one of the most commonly employed experimental techniques for the in situ or operando investigation of (electro)catalytically active interfaces as IR vibrational spectra provide a wealth of information about adsorbate species and their physicochemical interactions, such as chemical bonding, molecular orientation, or site-selective reactivity. ${ }^{1}$ While the electrochemical surface-enhanced variant of the technique, electrochemical surface-enhanced infrared absorption spectroscopy (EC-SEIRAS), achieves excellent signal-to-noise ratios even from (sub)monolayer adsorbates under potential control of the substrate,,$^{2,3}$ unambiguous spectral interpretation is often complicated by the joint occurrence of external field- or charge-induced Stark-type effects and the physico-chemical properties intrinsic to the system under study. Disentangling these different effects would be invaluable for an optimal exploitation of EC-SEIRAS data and provide helpful input for improved modeling approaches to enhance our fundamental understanding of (potentio-)dynamic interfacial electrochemical processes.

One prominent example for such unresolved spectral behavior is the drastic upshift by about 60 to $100 \mathrm{~cm}^{-1} / \mathrm{V}$ of the sulfate/Au(111) $v_{\mathrm{SO}}$ stretch vibration observed as a function of applied potential, E. ${ }^{4-6}$ Despite the fact that the sulfate/Au electrochemical system has been studied in great detail, ${ }^{7-9}$ questions about the fundamental nature of the potential-dependent molecule-substrate and molecule-molecule interactions have remained unresolved. ${ }^{10-13}$ Particularly, quantifying the extent to which an (external) Stark effect and/or coverage-dependent sulfate-sulfate (dipole) interactions contribute to the peak shift would 
provide important insight into the interfacial (re)activity of this important electrochemical model system but has not yet been achieved.

The coverage dependent contribution to the shift, $\Delta \tilde{v}(\Theta)$, can be ascribed to an increased Coulomb repulsion or dipoledipole coupling ${ }^{14}$ between neighboring sulfate anions as the sulfate surface coverage increases with time and increasing potential until the onset of Au surface oxidation. This enhanced lateral Coulomb repulsion compresses neighboring adsorbed sulfate anions and thereby strengthens the SO molecular bond, resulting in a $v_{\text {SO }}$ band upshift. In contrast, the Stark shift, $\Delta \tilde{v}(\phi)$, is based on the surface electric field, $\Phi$ (which is directly related to the applied potential, E) that causes the vibrational energy levels of the adsorbate to shift linearly with $\Delta \Phi .^{12,15,16}$ Furthermore, the downshift of the Fermi-level of the Au electrode with increasing positive potential relative to the filled anti-bonding states of the sulfate leads to backdonation of electrons from the anti-bonding states to the $\mathrm{Au}$, thereby strengthening the SO bond and upshifting the $v_{\mathrm{SO}}$ band. ${ }^{0,11,13}$

Note that for systems that exhibit a broad potential window with predefined stable adsorbate coverage, determining the Stark effect independently from changes in adsorbate coverage is rather straightforward: for example, Kunimatsu et al. determined the coverage and the Stark effect contributions to the overall $v_{\mathrm{CO}}$ band shift for $\mathrm{CO}$ on smooth Au electrodes in alkaline media to be 50 to $60 \mathrm{~cm}^{-1} / \theta_{\mathrm{CO}}$ and about $64 \mathrm{~cm}^{-1} / \mathrm{V}$, respectively. ${ }^{17}$ The Weaver group varied the $\mathrm{CO}$ coverage on $\mathrm{Pt}(111)$ by partial $\mathrm{CO}$ electrooxidation and found a $v_{\mathrm{CO}}$ peak shift of roughly $10 \mathrm{~cm}^{-1} / \theta_{\mathrm{CO}}$ for varying $\mathrm{CO}$ coverages. ${ }^{18}$ For the $\Delta \tilde{v}(\phi)$ Stark contribution, the same authors observed shifts of 34 to $44 \mathrm{~cm}^{-1} / \mathrm{V}$, depending on the CO coverage.

For sulfate adsorption on $\mathrm{Au}(111)$, however, the task to pinpoint and disentangle the influence of adsorbate coverage on the overall peak shift is challenging because saturated sulfate coverage is only reached within a narrow potential window. A different experimental approach to the usual $\mathrm{CO} /$ metal studies is thus required. Here, we propose a strategy to disentangle the total potential-dependent $v_{\text {SO }}$ band shift of $93.5 \pm 1.5 \mathrm{~cm}^{-1} / \mathrm{V}$ based on EC-SEIRAS potential-jump experiments that allow us to slow down the sulfate adsorption kinetics sufficiently to disentangle coverage from Stark effects, combined with cyclic voltammetry (CV). We derive a linear equation that contains both coverage dependence and Stark effect and allows us to extract numerical values to compare with the experimental data. As a result, we quantify the Stark effect to be $75.6 \pm 2.7 \mathrm{~cm}^{-1} / \mathrm{V}$, while the coverage increase accounts for $15.6 \pm 1.2 \mathrm{~cm}^{-1} / \theta_{\mathrm{SO}}$ of the total band shift. This detailed insight into the relationship between sulfate surface coverage, macroscopic electric field, and ECSEIRAS peak position demonstrates the great potential that our approach entails to enhance our fundamental understanding of the interplay of adsorbates at electrified solid-liquid interfaces.

\section{EXPERIMENTAL}

The EC-SEIRAS samples were $25 \mathrm{~nm}$-thick gold thin films (99.999\%, Kurt J. Lesker Ltd.) thermally evaporated on one of the faces of a low oxygen-content Si 60-prism (Pastec Ltd, Japan). Deposition was carried out in the vacuum chamber of a PVD75 coating system (Kurt J. Lesker Ltd.) at a base pressure of around $10^{-6}$ Torr using a quartz crystal microbalance to control both the thickness of the Au film and the deposition rate $(0.06 \AA / \mathrm{s})$. Once in the spectro-electrochemical cell, the $\mathrm{Au}$ thin film electrodes were cleaned by applying a few voltammetric cycles up to the onset of surface oxidation in aqueous $0.1 \mathrm{M} \mathrm{H}_{2} \mathrm{SO}_{4}$ solution.

EC-SEIRAS experiments in an ATR configuration were performed with a Nicolet 8700 (Thermo Scientific) spectrometer controlled by OMNIC software and equipped with a liquid$\mathrm{N}_{2}$-cooled MCT-A detector and a wire grid ZnSe polarizer (Pike Tech.). The spectro-electrochemical cell equipped with the EC-SEIRAS sample was placed at the top of a Veemax reflectance accessory (Pike Tech.). All EC-SEIRA spectra were collected with a resolution of $4 \mathrm{~cm}^{-1}$ and are plotted in absorbance units, $A$, which are defined as $A=-\log \left(I / I_{\text {ref }}\right)$, where $I$ is the intensity of the reflected light at the sample surface and $I_{r e f}$ is the corresponding intensity at the reference potential of $0.1 \mathrm{~V}$ vs. the reversible hydrogen electrode (RHE). The reference spectra at $0.1 \mathrm{~V}$ vs. RHE are recorded in a potential region where (specific) anion adsorption is negligible. Positive and negative EC-SEIRAS bands correspond to the gain or loss of species, respectively, at the sample potential with respect to the reference potential. The $v_{\text {So }}$ peak area was normalized to the mean value of the extracted $v_{\text {SO }}$ peak area from 20 spectra recorded between 1.15 and $1.3 \mathrm{~V}$ vs. RHE where the peak area reaches saturation. At saturation, sulfate ions are known to cover approximately $20 \%$ of the $\mathrm{Au}(111)$ surface, ${ }^{5}$ denoted as saturation coverage, $\theta_{\mathrm{SO}}$, in this manuscript.

All glassware and Teflon cell parts were cleaned by boiling them in $\mathrm{HNO}_{3}$ with subsequent rinsing-boiling cycles in ultrapure water (18.2 M $\Omega \mathrm{cm}$, TOC $50 \mathrm{ppb}$ max, Elga Vivendi). As a reference electrode, a trapped hydrogen electrode (Pt wire with the $\mathrm{H}_{2}$ reservoir (RHE); all potentials are reported vs. RHE) was used and freshly prepared directly before the experiments. A gold wire was used as a counter electrode. The potentiostat Model 175 Universal Programmer (Princeton Applied Research) with the eDAQ e-corder with Chart software was used for potential control. Electrolyte solutions $(0.1$ $\mathrm{M} \mathrm{H}_{2} \mathrm{SO}_{4}$ ) were prepared from $\mathrm{H}_{2} \mathrm{SO}_{4}$ (Merck Suprapur, 96\%) and ultrapure water. Ar gas (N50, Air Liquide) was used to deaerate the working solutions and to blanket them during the experiments. The stability of the Au thin films was periodically examined by checking for visible macroscopic changes of the Au film, mutations of the recorded CVs and sudden spectral changes in the slope toward high wavenumbers. The Au thin films exhibited the order/disorder transition peak around $1.1 \mathrm{~V}$ vs. RHE proving to be quasi-Au(111)-like and referred to as $\mathrm{Au}(111)$ in the following. For all EC-SEIRAS measurements, interferograms with an acquisition time of $350 \mathrm{~ms}$ were recorded. The reference spectrum consisted of an average of 
90 spectra recorded at $0.1 \mathrm{~V}$ vs. RHE directly before each measurement. For the fitting of the spectral data, performed in Matlab, a Gaussian line shape and linear baseline subtraction were used.

\section{RESULTS AND DISCUSSION}

\section{Continuous scan experiments}

Figure 1(a) shows the normalized peak area of the sulfate band $v_{\text {SO }}$ at around $1200 \mathrm{~cm}^{-1}$ as a function of applied potential for the up-scan to more positive potentials (black) and downscan to more negative potentials (green) as well as the simultaneously acquired CV (gray) (see Fig. S1 of the supplementary material for an example EC-SEIRA spectrum). Characterizing the quality of our $\mathrm{Au}(111)$ surface, from the $\mathrm{CV}$, we obtain a surface charge density for the Au oxidation/reduction and sulfate adsorption/desorption of $1.41 \mathrm{mC} / \mathrm{cm}^{2}$ and of $0.134 \mathrm{mC} / \mathrm{cm}^{2}$ for the double layer charging, which indicates quasi-Au(111) surface of less than a factor two roughness compared to ideal $\mathrm{Au}(111)$ (see Ref. 19 and Fig. S2 of the supplementary material for details).

The $v_{\text {SO }}$ peak starts to grow at around $0.6 \mathrm{~V}$ vs. RHE and saturates between 1.15 and $1.3 \mathrm{~V}$ vs. RHE. Above $1.35 \mathrm{~V}$ vs. RHE, coinciding with the onset of Au surface oxidation, the $v_{\mathrm{SO}}$ peak decreases and vanishes at around $1.55 \mathrm{~V}$ vs. RHE. During the potential down-scan to more negative potentials, the $v_{\text {SO }}$ peak area starts to increase at approximately $1.2 \mathrm{~V} v \mathrm{vs}$. RHE. The hysteresis between the peak appearance in upward and downward scan directions has been observed previously ${ }^{20}$ and can be explained by the fact that before sulfate adsorption, $\mathrm{Au}$ oxide needs to be reduced to free $\mathrm{Au}$ sites, consequently delaying sulfate re-adsorption in the downward scan direction (sweep to more negative potentials). Note that we do not observe any spectral features that can be associated with sulfate adsorption on $\mathrm{Au}$ oxide. We speculate that this absence of additional spectral bands indicates the occurrence of a second point of zero charge (pzc) for sulfate adsorption on $\mathrm{Au}$ oxide at a potential of around $1.35 \mathrm{~V}$, similar to what has been suggested by the Eikerling group for Pt (oxide). ${ }^{21,22}$ The peak area reaches its maximum at around $1.1 \mathrm{~V}$ vs. RHE before dropping continuously until reaching noise level at $\sim 0.6 \mathrm{~V}$ vs. RHE. As the recorded EC-SEIRAS peak intensities exhibit the same potential-dependence as chronocoulometric and radiotracer data that have been previously used to assess sulfate surface coverages [Ref. 20 and Fig. S3 of the supplementary material], we can employ the peak area as a direct measure for the sulfate surface coverage. In agreement with the literature, effects of potential-dependent surface enhancement ${ }^{23}$ or molecular reorientation ${ }^{4}$ on the band intensities can be excluded.

In Fig. 1(b), the $v_{\text {SO }}$ peak position is plotted as a function of applied potential for the scan to more positive potentials (black) and more negative potentials (green). The complete upward scan and the downward scan from $\sim 1.1 \mathrm{~V}$ vs. RHE (after a sharp increase in peak position between 1.1 and $1.2 \mathrm{~V}$ vs. RHE due to the fast recovery of the sulfate saturation coverage) exhibit similar linear behavior and can be fitted with a slope of $\sim 93.5 \pm 1.5 \mathrm{~cm}^{-1} / \mathrm{V}$. This potential-dependent shift, $\Delta \tilde{v}(E)$, is close to the ones previously reported by other groups of 60 to $100 \mathrm{~cm}^{-1} / \mathrm{V} .^{4-6}$ It contains contributions from both the field-induced Stark effect and coverage-dependent adsorbate-adsorbate dipole interactions that cannot be easily disentangled from the EC-SEIRAS data of the continuous scan experiment. If one assumes that complete saturation coverage is reached in the potential range between 1.15 and $1.3 \mathrm{~V}$ vs. RHE (blue highlighted region in Fig. 1), one can fit the corresponding region in Fig. 1(b) separately (Fig. S4 of the supplementary material). In principle, the so obtained slope of $75.6 \pm 2.7$ $\mathrm{cm}^{-1} / \mathrm{V}$ should depict the pure Stark shift contribution, $\Delta \tilde{v}(\phi)$, to the total shift. As the number of data points in this region is rather limited and the assumption of full coverage may not be perfectly valid given the large scattering of data points in this region, in the following, we suggest an additional, more precise experimental approach to separate the two effects.

\section{Potential-jump experiments}

To obtain the pure coverage dependent contribution, $\Delta \tilde{v}(\Theta)$, to the total potential-induced shift, we performed potential-jump experiments. The underlying idea of this approach is based on two observations: (i) The sulfate surface coverage starts to decrease with the onset of Au oxidation and recovers when the Au oxide is reduced as sulfate ions do not adsorb on $\mathrm{Au}$ oxide but only on pure Au. (ii) The reduction of Au oxide around the reduction onset potential of $\sim 1.2 \mathrm{~V}$ vs. RHE is much slower, i.e., in the order of a few seconds, than the kinetics of sulfate adsorption on Au. We can thus
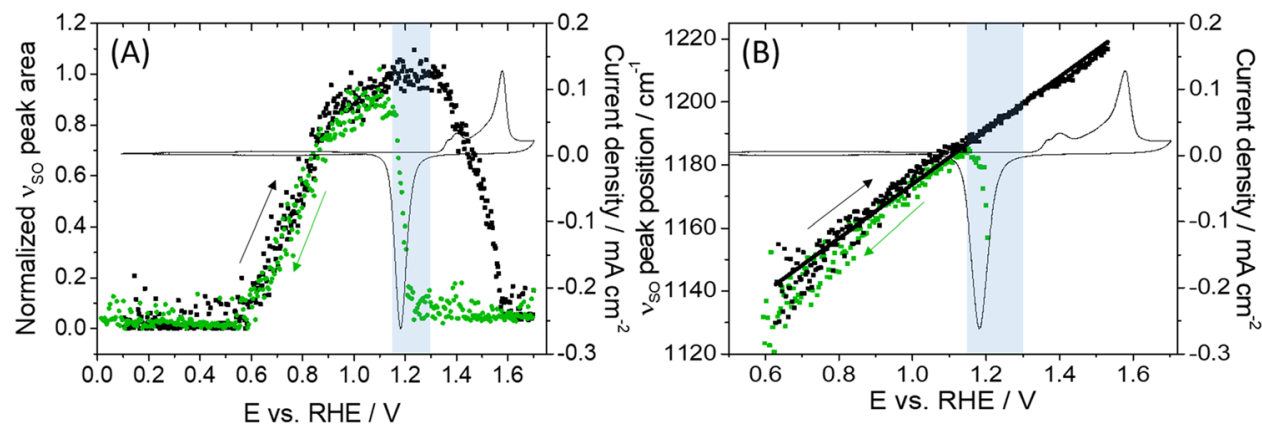

FIG. 1. (a) Normalized $v_{S O}$ peak area plotted as a function of potential and (b) $v_{S O}$ peak position plotted as a function of potential during $\mathrm{CV}$ in upward (to more positive potentials, black) and downward (to more negative potentials, green) scan direction at $10 \mathrm{mV} / \mathrm{s}$ scan rate (electrolyte: $0.1 \mathrm{M} \mathrm{H}_{2} \mathrm{SO}_{4}$ ); simultaneously recorded CV (gray). The solid black line in (b) is a linear fit to the data. The blue highlighted region indicates the potential region of approximate saturation sulfate coverage. 

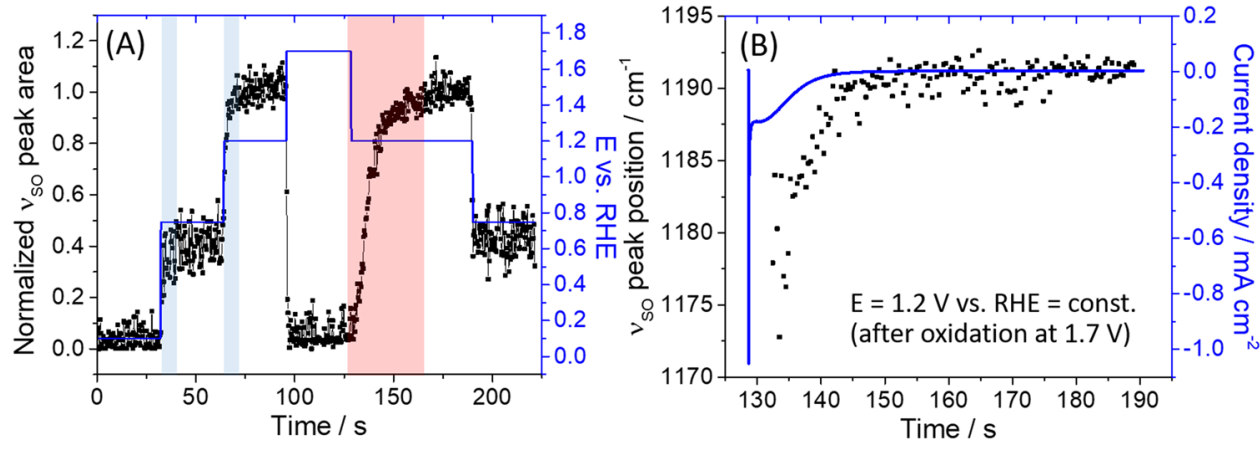

FIG. 2. (a) Normalized $v_{\text {so }}$ peak area (black) and applied potentials (blue) plotted as a function of time. (b) $v_{\text {so }}$ peak position (black) and current trace (blue) as a function of time at a fixed potential of $1.2 \mathrm{~V}$ vs. RHE after prior surface oxidation at $1.7 \mathrm{~V}$ vs. RHE (electrolyte: $0.1 \mathrm{M}$ $\mathrm{H}_{2} \mathrm{SO}_{4}$ ). Spectral (data point) acquisition time $=350 \mathrm{~ms}$ slowly reduce the oxidized Au surface at a constant potential of $1.2 \mathrm{~V}$ vs. RHE to create a free Au surface to slow down the sulfate coverage increase. This strategy allows us to monitor the effect of the coverage change during sulfate adsorption at a fixed (surface) potential, i.e., with a "fixed" Fermi level, in this way disentangling the contributions of coverage dependence and Stark effect to the total potential-dependent band shift.

The proposed strategy was implemented by applying a potential-jump sequence of $0.1 \rightarrow 0.75 \rightarrow 1.2 \rightarrow 1.7 \rightarrow 1.2$ $\rightarrow 0.75 \mathrm{~V}$ vs. RHE to the Au electrode while recording EC-SEIRA spectra (Fig. S5 of the supplementary material). Each potential was held for 32 to $64 \mathrm{~s}$ before jumping to the next potential value. Figure 2(a) shows the normalized $v_{\text {SO }}$ peak area (black data points) and the respective potential steps (blue line) as a function of time for an example data set. The regions highlighted in light blue (upward jumps of $0.1 \rightarrow 0.75 \rightarrow 1.2 \mathrm{~V}$ vs. RHE) show an immediate rise in the $v_{\text {SO }}$ peak area due to the fast adsorption of sulfate to Au. The light red region (downward jump of $1.7 \rightarrow 1.2 \mathrm{~V}$ vs. RHE) shows a slow rise of the $v_{\text {SO }}$ peak area as the Au oxide formed at $1.7 \mathrm{~V}$ vs. RHE has to be reduced before sulfate adsorption can proceed, in this way providing a sufficient number of data points for analysis. As sulfate adsorption on Au proceeds, a shift in $v_{\text {SO }}$ peak position to higher wavenumbers from about 1173 to $1191 \mathrm{~cm}^{-1}$ is observed until $\theta_{\mathrm{SO}}$ is reached [Fig. 2(b), black data points]. Figure 2(b) also shows the simultaneously recorded current vs time trace (blue line) recorded after stepping the potential from 1.7 to $1.2 \mathrm{~V}$ vs. RHE. The integration of the current curve gives a total charge of $1.45 \mathrm{mC} / \mathrm{cm}^{2}$, which is in good agreement with the above-obtained values from $\mathrm{CV}$. The current decays exponentially within the first couple of seconds due to the fast onset of $\mathrm{Au}$ oxide reduction that is followed by a slower decay over $\sim 15 \mathrm{~s}$. Note that the sulfate $v_{\text {SO }}$ peak in the potential-jump EC-SEIRA spectra is only observed after the first fast current decay.

In Fig. 3, we plot the $\boldsymbol{v}_{\mathrm{SO}}$ peak positions vs the respective normalized peak areas to summarize the results from potential-jump (black) and continuous scan (gray) experiments as displayed in Figs. 1 and 2. The data from the potentialjump experiments show a linear increase in peak position with increasing peak area. As the jump experiments are performed at a fixed potential, the Stark effect contribution must be assumed to be constant throughout the investigated time window for all surface coverages (i.e., peak areas) until sulfate saturation coverage, $\theta_{\mathrm{SO}}$. The potential drop across the bare gold-electrolyte interface is set immediately, as suggested by the immediate rise in $v_{\text {SO }}$ peak area prior to oxidation [Fig. 2(a), regions highlighted in blue]. From the slopes of three independent potential-jump experiments (Fig. S6 of the supplementary material), we quantify the (average) pure coverage-dependent peak shift (i.e., the peak shift due to Coulomb repulsion and dipole-dipole interactions between the molecules within the surface adlayer), $\Delta \tilde{\nu}(\Theta)$, to be 15.6 $\pm 1.2 \mathrm{~cm}^{-1} / \theta_{\mathrm{SO}}$. The average peak positions reached at $\theta_{\mathrm{SO}}$ of $1191 \mathrm{~cm}^{-1}$ converge at a potential of $E=1.2 \mathrm{~V}$ vs. RHE (right $\mathrm{y}$-axis, with the correlation between peak position and applied potential taken from Fig. 1) as expected.

On the other hand, the collected continuous scan data points (gray) show a peak position upshift in potential regions where the sulfate coverage increases (at potentials between 0.5 and $1.3 \mathrm{~V}$ vs. RHE, as indicated on the right $y$-axis) as well as for potential regions where the sulfate coverage decreases (at potentials between 1.3 and $1.55 \mathrm{~V}$ vs. RHE), amounting to a total shift of $93.5 \pm 1.5 \mathrm{~cm}^{-1} / \mathrm{V}$ as stated above. The nonlinear trace confirms that the total EC-SEIRAS peak shift observed during CV cannot be solely explained by potential-induced sulfate

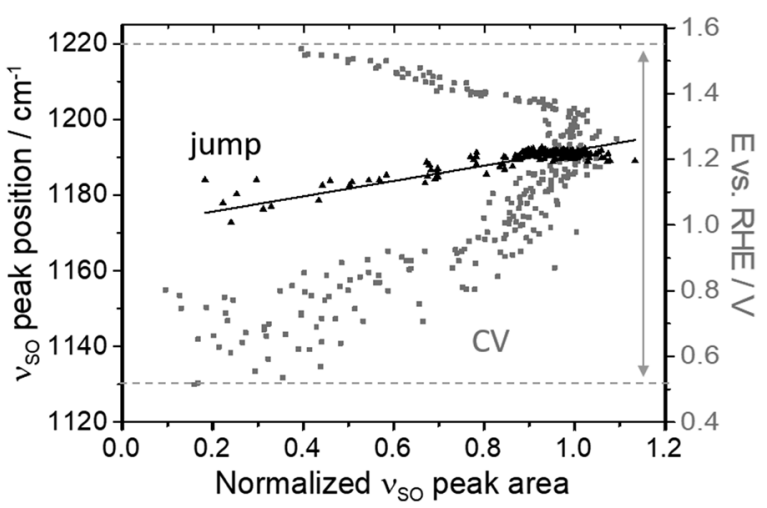

FIG. 3. Peak position vs peak area plot for data points obtained from potentialjump (black) and continuous-scan CV (gray) EC-SEIRAS experiments (in $0.1 \mathrm{M}$ $\mathrm{H}_{2} \mathrm{SO}_{4}$ ) described in Figs. 1 and 2. The right $y$-axis notes the respective potentials during CV EC-SEIRAS. 
coverage change. Note that for lack of spectral evidence, we neglect possible (dipole) interactions between Au oxide and adsorbed sulfate anions at potentials between 1.3 and $1.55 \mathrm{~V}$ vs. RHE.

\section{Modeling of the peak shift}

Figures 1(b) and 3 suggest, to a good first approximation, a linear dependence of the sulfate peak position on the applied potential and on the sulfate coverage, respectively. Therefore, the sulfate peak position, $\tilde{\nu}_{S O}$, has to depend linearly on both the surface potential, $\Phi$ (which is directly related to the applied potential $E$ ), and on the sulfate surface coverage (which implicitly depends on $\Phi$ and on time, $t), \theta(\Phi, t)$, and can be expressed by the following equation:

$$
\tilde{v}_{\mathrm{SO}}(\phi, \Theta)=\mathrm{K}_{\phi}\left(\phi-\phi_{0}\right)+\mathrm{K}_{\Theta} \Theta+\tilde{v}_{0},
$$

where $K_{\Phi}$ is the slope-constant associated with the Stark effect and $K_{\theta}$ is the slope-constant associated with the sulfate surface coverage, and $\tilde{v}_{0}$ is the (hypothetical) vibrational SO stretch frequency of an adsorbed, isolated sulfate molecule (which in earlier studies has been denoted as singleton ${ }^{14}$ ) at $\Phi_{0}$, the potential at which sulfate adsorption starts. From Fig. 1(b), we can extract $\Phi_{0}$ to be $0.56 \mathrm{~V}$ and $\tilde{v}_{0}$ to be 1136 $\mathrm{cm}^{-1}$. It is interesting to note that while there is a sulfate band observed at potentials lower than those in which the linear relation is observed in Fig. 1(b), the linear extrapolation of the peak position leads to a value so close to that from the differential capacity in the absence of any specific adsorption, i.e., to the pzc of $\mathrm{Au}(111){ }^{24,25}$

In the following, we test Eq. (1) for consistency with the experimental results: Can the coverage dependence be decoupled from all other potential-dependent effects in this particular form? We are in particularly interested in the comparison of $K_{\theta}$ extracted from CV and jump experiments, respectively, as additional verification of the proposed jump approach. For the CV EC-SEIRAS data [varying the (surface) potential $\Phi$ and $\theta]$, the total derivative in $\Phi$ of Eq. (1) can be determined as

$$
\frac{d \tilde{v}}{d \phi}=\frac{\partial \tilde{v}}{\partial \phi} \cdot \frac{d \phi}{d \phi}+\frac{\partial \tilde{v}}{\partial \theta} \cdot \frac{d \theta}{d \phi}=K_{\phi}+K_{\theta} \cdot \frac{d \theta}{d \phi} .
$$

The analysis of derivatives is convenient as the explicit function of $\theta(\Phi, t)$ is unknown. cases

Within this framework, let us consider the three limiting

(i) If $\theta$ does not change, $\frac{d \theta}{d \phi}=0$, Eq. (2) can be simplified to $\frac{d \tilde{v}}{d \phi}=K_{\phi}$. To determine the constant $K_{\Phi}$, we identify the potential range in which $\theta$ is approximately saturation, i.e., $\frac{d \theta}{d \phi} \approx 0$ (Fig. 1). As already briefly described above, within this potential range of 1.15 to $1.3 \mathrm{~V}$ vs. RHE, a linear fit of the $v_{\text {SO }}$ peak position as a function of potential reveals a slope $\frac{d \hat{v}}{d \phi}=K_{\phi}$ of $75.6 \pm 2.7 \mathrm{~cm}^{-1} / \mathrm{V}$.

(ii) For $\frac{d \theta}{d \phi}>0$, a positive slope is found in the potential range from 0.63 to $0.95 \mathrm{~V}$ vs. RHE [Fig. 1(a)] that in a first approximation can be viewed as linear, i.e., $\frac{d \tilde{v}}{d \phi}=$ const. From Fig. 1(b), a linear fit of the $v_{\text {So }}$ peak position as a function of potential in the range from 0.63 to $0.95 \mathrm{~V}$ vs. RHE returns

$$
\frac{d \tilde{v}}{d \phi}=K_{\phi}+K_{\theta} \cdot \frac{d \theta}{d \phi}=105.5 \pm 5.1 \frac{\mathrm{cm}^{-1}}{\mathrm{~V}} .
$$

Solving for $\mathrm{K}_{\theta}$ leads to the following expression:

$$
K_{\theta}=\left(105.5 \frac{\mathrm{cm}^{-1}}{\mathrm{~V}}-\mathrm{K}_{\phi}\right) \cdot \frac{d \phi}{d \theta} .
$$

From Fig. 1(a), $\frac{d \theta}{d \phi}$ can be determined within the potential range from 0.63 to $0.95 \mathrm{~V}$ vs. RHE with another linear fit and amounts to $2.43 \pm 0.07 \theta_{\mathrm{SO}} / \mathrm{V}$. The reciprocal, $\frac{d \phi}{d \theta}$, is $0.41 \pm 0.01 \mathrm{~V} / \theta_{\text {SO }}$. Inserting $K_{\Phi}$ and $\frac{d \phi}{d \theta}$ in Eq. (4), $K_{\theta}$ amounts to $12.3 \pm 3.5 \mathrm{~cm}^{-1} / \theta_{\mathrm{SO}}$ (see the supplementary material for error propagation). This extracted value of $K_{\theta}$ matches sufficiently the corresponding value extracted from the potential-step experiments $(15.6 \pm$ $\left.1.2 \mathrm{~cm}^{-1} / \theta_{\mathrm{SO}}\right)$ and confirms the validity of the proposed linear Eq. (1).

(iii) For $\frac{d \theta}{d \phi}<0$, i.e., in the potential range from 1.3 to $1.53 \mathrm{~V}$ vs. RHE in Fig. 1(a), a similar analysis as for case (ii) returns an error-prone $K_{\theta}$ of $0.04 \pm 1.7 \mathrm{~cm}^{-1} / \theta_{\mathrm{SO}}$ (see the supplementary material for details). The analysis is jeopardized by the fact that $\mathrm{Au}(111)$ oxidation starts first at defect sites before it proceeds to (111)terrace sites, leading to spatially inhomogeneous sulfate desorption that cannot be adequately described with a simple linear model.

According to Eq. (1), also the total derivative in $\theta$ can be further determined as

$$
\frac{d \tilde{v}}{d \theta}=\frac{\partial \tilde{v}}{\partial \phi} \cdot \frac{d \phi}{d \theta}+\frac{\partial \tilde{v}}{\partial \theta} \cdot \frac{d \theta}{d \theta}=K_{\phi} \cdot \frac{d \phi}{d \theta}+K_{\theta} .
$$

For the potential-jump experiments (varying $\theta$, constant $\Phi$ ), Eq. (5) simplifies to $\frac{\partial \hat{v}}{\partial \theta}=K_{\theta}$. This notation suggests a linear trend for $\tilde{v}(\Phi=$ const., $\theta)$ which is indeed observed experimentally (Fig. 3). From the potential-jump experiments, $K_{\theta}$ amounts to $15.6 \pm 1.2 \mathrm{~cm}^{-1} / \theta_{\mathrm{SO}}$, which, as already pointed out above, matches well the $K_{\theta}$ value of $12.3 \pm 3.5 \mathrm{~cm}^{-1} / \theta_{\mathrm{SO}}$ extracted from Eq. (2) using the CV EC-SEIRAS data. As it captures all experimental observations adequately, Eq. (1) can be evaluated as an effective descriptor for decoupling the sulfate coverage dependence from the Stark effect contributions to the overall observed potential-dependent peak shift. The individual values for the peak shift contributions are summarized in Table I.

Note that while this simple linear treatment describes the experimental observations with surprising accuracy and allows for direct quantification of adsorbate-adsorbate and adsorbate-field interactions, a more refined modeling including potential-induced $\mathrm{Au}$ oxide or hydroxide formation/dissolution, water displacement and/or reorientation, 
TABLE I. Summary of peak shift contributions.

Total potential-dependent shift

Stark effect contribution

Coverage dependence contribution

(potential jump)

Coverage dependence contribution

[Eq. (2)]

$$
\begin{gathered}
\mathrm{K}_{\mathrm{E}}=93.5 \pm 1.5 \mathrm{~cm}^{-1} / \mathrm{V} \\
\mathrm{K}_{\Phi}=75.6 \pm 2.7 \mathrm{~cm}^{-1} / \mathrm{V} \\
\mathrm{K}_{\theta}=15.6 \pm 1.2 \mathrm{~cm}^{-1} / \theta_{\mathrm{SO}} \\
\mathrm{K}_{\theta}=12.3 \pm 3.5 \mathrm{~cm}^{-1} / \theta_{\mathrm{SO}}
\end{gathered}
$$

and the thermodynamic specifics of heterogeneous adsorption sites would be required to increase the accuracy and transferability of the model.

\section{SUMMARY}

To summarize, we have investigated the potentialinduced EC-SEIRAS $v_{\text {SO }}$ band shift of $93.5 \pm 1.5 \mathrm{~cm}^{-1} / \mathrm{V}$ of sulfate/Au(111) in $0.1 \mathrm{M} \mathrm{H}_{2} \mathrm{SO}_{4}$ in terms of coverage and Stark effect contributions. To assess the pure contribution of sulfate coverage increase to the peak upshift, we have performed potential-jump experiments at a fixed potential, i.e., Stark tuning. Our approach takes advantage of the slow reduction kinetics of $\mathrm{Au}$ oxide compared to (fast) sulfate adsorption at the reduction onset at around $1.2 \mathrm{~V}$ vs. RHE. This experimental strategy allowed us to quantify the coverage contribution to the overall peak shift to be in the order of $15.6 \pm 1.2 \mathrm{~cm}^{-1} / \theta_{\mathrm{SO}}$ by a linear fit of the $v_{\text {SO }}$ peak position versus peak intensity (i.e., sulfate coverage) plot. We have presented and tested a linear model for the peak shift to extract the contribution of the field-induced Stark effect. The derivative analysis returns reasonable estimates for the Stark shift of $75.6 \pm 2.7 \mathrm{~cm}^{-1} / \mathrm{V}$ and for the coverage contribution of about $12.3 \pm 3.5 \mathrm{~cm}^{-1} / \theta_{\mathrm{SO}}$. As such, about $15 \%$ of the total shift within the investigated potential range can be attributed to sulfate-sulfate (dipole) interactions, while the remaining $85 \%$ are due to field-induced Stark effects.

Evidently, even well-defined electrochemical adsorbate model systems like sulfate/Au(111) that have been studied in great depth for a prolonged time display a high level of complexity in their fundamental potential-dependent behavior whose precise nature is still far from being completely understood. As demonstrated here, spectro-electrochemical methods like EC-SEIRAS provide an enormous potential to help elucidate molecular interactions and field-dependent responses of interfacial layers at electrified surfaces by providing direct quantitative insights about (re)active adsorbates under realistic working conditions that can furthermore be employed as reliable input for advanced simulation approaches.

\section{SUPPLEMENTARY MATERIAL}

See supplementary material for example EC-SEIRA spectra for CV and jump experiments, CV electrode characterization, relation between Gibbs excess and SEIRAS intensity, linear data fittings and evaluation including error propagation of the derivative analysis.

\section{ACKNOWLEDGMENTS}

J.H.K.P. and K.F.D. gratefully acknowledge financial support by the Max Planck Graduate Center with the Johannes Gutenberg University Mainz (MPGC). U.E.Z. is grateful for financial support from the Alexander von Humboldt Foundation. J.M.F. thanks MCINN-FEDER (Spain) for support through Project No. CTQ2016-76221-P. K.F.D. acknowledges generous support through the Emmy Noether Program of the Deutsche Forschungsgemeinschaft (No. DO1691/1-1) and through the "Plus 3" Program of the Boehringer Ingelheim Foundation. We are thankful to Valentín Briega-Martos, Maksim Grechko, Bogdan Marekha, and Ivan Ivanov for support and valuable discussions.

The authors declare no competing financial interests.

\section{REFERENCES}

${ }^{1}$ F. Zaera, Chem. Soc. Rev. 43, 7624 (2014).

${ }^{2}$ M. Osawa, Bull. Chem. Soc. Jpn. 70, 2861 (1997).

${ }^{3}$ A. Rodes, J. M. Orts, J. M. Pérez, J. M. Feliu, and A. Aldaz, Electrochem. Commun. 5, 56 (2003).

${ }^{4} \mathrm{~K}$. Ataka and M. Osawa, Langmuir 14, 951 (1998).

${ }^{5}$ G. J. Edens, X. Gao, and M. J. Weaver, J. Electroanal. Chem. 375, 357 (1994).

${ }^{6}$ U. Zhumaev, A. V. Rudnev, J. F. Li, A. Kuzume, T. H. Vu, and T. Wandlowski, Electrochim. Acta 112, 853 (2013).

${ }^{7}$ O. M. Magnussen, J. Hageböck, J. Hotlos, and R. J. Behm, Faraday Discuss. 94, 329 (1992).

${ }^{8}$ Y. Shingaya and M. Ito, J. Electroanal. Chem. 467, 299 (1999).

${ }^{9}$ T. Wandlowski, K. Ataka, S. Pronkin, and D. Diesing, Electrochim. Acta 49, 1233 (2004).

${ }^{10}$ P. W. Faguy, N. Markovic, R. R. Adzic, C. A. Fierro, and E. B. Yeager, J. Electroanal. Chem. 289, 245 (1990).

${ }^{11}$ E. M. Patrito, P. Paredes Olivera, and H. Sellers, Surf. Sci. 380, 264 (1997).

${ }^{12}$ D. K. Lambert, Electrochim. Acta 41, 623 (1996).

${ }^{13}$ K. Ataka, T. Yotsuyanagi, and M. Osawa, J. Phys. Chem. 100, 10664 (1996).

${ }^{14}$ P. Hollins and J. Pritchard, Prog. Surf. Sci. 19, 275 (1985).

${ }^{15}$ D. M. Bishop, J. Chem. Phys. 98, 3179 (1993).

${ }^{16}$ R. Jinnouchi, T. Hatanaka, Y. Morimoto, and M. Osawa, Electrochim. Acta 101, 254 (2013).

${ }^{17}$ K. Kunimatsu, A. Aramata, N. Nakajima, and H. Kita, J. Electroanal. Chem. Interfacial Electrochem. 207, 293 (1986).

${ }^{18}$ L. W. H. Leung, A. Wieckowski, and M. J. Weaver, J. Phys. Chem. 92, 6985 (1988).

${ }^{19}$ E. Herrero, J. M. Feliu, A. Wieckowski, and J. Clavilier, Surf. Sci. 325, 131 (1995).

${ }^{20}$ Z. Shi, J. Lipkowski, M. Gamboa, P. Zelenay, and A. Wieckowski, J. Electroanal. Chem. 366, 317 (1994).

${ }^{21}$ J. Huang, A. Malek, J. Zhang, and M. H. Eikerling, J. Phys. Chem. C 120, 13587 (2016).

22 J. Huang, T. Zhou, J. Zhang, and M. Eikerling, J. Chem. Phys. 148, 044704 (2018).

${ }^{23}$ M. Zapata Herrera, J. Aizpurua, A. K. Kazansky, and A. G. Borisov, Langmuir 32, 2829 (2016).

${ }^{24}$ D. M. Kolb and J. Schneider, Electrochim. Acta 31, 929 (1986).

${ }^{25}$ B. Álvarez, V. Climent, J. M. Feliu, and A. Aldaz, Electrochem. Commun. 2, 427 (2000). 